\title{
Getting the best out of T2K and NOvA
}

\section{Suprabh Prakash ${ }^{* \dagger}$}

Indian Institute of Technology Bombay, Mumbai

E-mail: suprabh@iitb.ac.in

\section{Sushant K. Raut}

Physical Research Laboratory, Ahmedabad

E-mail: sushant@prl.res.in

\section{S. Uma Sankar}

Indian Institute of Technology Bombay, Mumbai

E-mail: uma@phy.iitb.ac.in

We explore the combined physics potential of $\mathrm{T} 2 \mathrm{~K}$ and NOvA in light of the moderately large measured value of $\theta_{13}$. For $\sin ^{2} 2 \theta_{13}=0.1$, which is close to the best fit value, a $90 \%$ C.L. evidence for the hierarchy can be obtained only for the combinations (Normal hierarchy, $-170^{\circ} \leq \delta_{C P} \leq 0^{\circ}$ ) and (Inverted hierarchy, $0^{\circ} \leq \delta_{C P} \leq 170^{\circ}$ ), with the currently planned runs of NOvA and T2K. However, the hierarchy can essentially be determined for any value of $\delta_{C P}$, if the statistics of NOvA are increased by $50 \%$ and those of T2K are doubled. Such an increase will also give an allowed region of $\delta_{C P}$ around its true value, except for the CP conserving cases $\delta_{C P}=0$ or $\pm 180^{\circ}$.

36th International Conference on High Energy Physics

4-11 July 2012

Melbourne, Australia

\footnotetext{
* Speaker.

${ }^{\dagger}$ The speaker would like to thank the organisers and convenors of ICHEP 2012 and acknowledges the support provided during the conference.
} 


\section{Introduction}

The global fits to data from the accelerator experiments T2K [1] and MINOS [2] and the reactor experiments DChooz [3], Daya Bay [4] and RENO [5] have determined $\theta_{13}$ to be non-zero at $5 \sigma$ level, with the best fit very close to $\sin ^{2} 2 \theta_{13} \simeq 0.1[6,7]$. In light of these current and expected near future measurements, the next goals of neutrino oscillation experiments are the determination of neutrino mass hierarchy, detection of CP violation in the leptonic sector and measurement of $\delta_{C P}$. These goals can be achieved by high statistics accelerator experiments measuring $v_{\mu} \rightarrow v_{e}$ and $\bar{v}_{\mu} \rightarrow \bar{v}_{e}$ oscillation probabilities. In this paper, we study the combined ability of T2K and NOvA to achieve the above goals. For a detailed treatment of this same work, see [8].

\section{Simulation Details}

We use the software GLoBES $[9,10]$ for simulating the data of T2K and NOvA $[11,12,13$, $14,15,16,17,18,19]$. The basic properties of NOvA are taken from Ref. [14] and of T2K are taken from Ref. [11]. The efficiencies for each of the experiments are taken from GLoBES [9, 10].

We show the sensitivity of the experiments to hierarchy using the hierarchy exclusion plots. These are plotted in the plane of true values of $\sin ^{2} 2 \theta_{13}-\delta_{C P}$. The contours in these plots define the line $\chi^{2}=2.71$. In computing this $\chi^{2}$, we have marginalized over the neutrino oscillation parameter ranges. For all sets of parameter values to the right of the contour, the wrong hierarchy can be ruled out at $90 \%$ C.L. Throughout this paper, the phrase "hierarchy determination" implies $90 \%$ C.L. evidence for hierarchy.

\section{Hierarchy determination with $P_{\mu e}$}

In the presence of matter, the $v_{\mu} \rightarrow v_{e}$ oscillation probability, expanded perturbatively in the small mass-squared difference, $\Delta_{21}$ is given by [20, 21, 22]

$$
\begin{aligned}
P\left(v_{\mu} \rightarrow v_{e}\right)= & P_{\mu e}=\sin ^{2} 2 \theta_{13} \sin ^{2} \theta_{23} \frac{\sin ^{2} \hat{\Delta}(1-\hat{A})}{(1-\hat{A})^{2}} \\
& +\alpha \cos \theta_{13} \sin 2 \theta_{12} \sin 2 \theta_{13} \sin 2 \theta_{23} \cos \left(\hat{\Delta}+\delta_{C P}\right) \frac{\sin \hat{\Delta} \hat{A} \sin \hat{\Delta}(1-\hat{A})}{1-\hat{A}} \\
& +\alpha^{2} \sin ^{2} 2 \theta_{12} \cos ^{2} \theta_{13} \cos ^{2} \theta_{23} \frac{\sin ^{2} \hat{\Delta} \hat{A}}{\hat{A}^{2}}
\end{aligned}
$$

where $\hat{\Delta}=\Delta_{31} L / 4 E, \hat{A}=A / \Delta_{31}, \alpha=\Delta_{21} / \Delta_{31} . A$ is the Wolfenstein matter term [23] and is given by $A\left(\mathrm{eV}^{2}\right)=0.76 \times 10^{-4} \rho(\mathrm{gm} / \mathrm{cc}) E(\mathrm{GeV})$. For $\mathrm{NH}, \Delta_{31}$ is positive and for $\mathrm{IH}, \Delta_{31}$ is negative. The matter term $A$ is positive for neutrinos and is negative for anti-neutrinos. Hence, in neutrino oscillation probability, $\hat{A}$ is positive for $\mathrm{NH}$ and is negative for $\mathrm{IH}$. For anti-neutrinos, $\hat{A}$ is negative for $\mathrm{NH}$ and positive for $\mathrm{IH}$ and the sign of $\delta_{C P}$ is reversed.

The favourable and unfavourable half planes for a particular hierarchy can be defined from Eq. (3.1), where the $\delta_{C P}$ dependence occurs purely in the form $\cos \left(\hat{\Delta}+\delta_{C P}\right)$. If $\mathrm{NH}$ is the true hierarchy, $\hat{\Delta} \approx 90^{\circ}$ around the probability maximum. Then, the $\delta_{C P}$ dependent term increases $P_{\mu e}$ if $\delta_{C P}$ is in the lower half plane (LHP) and decreases it if $\delta_{C P}$ is in the upper half plane (UHP). 
Hence a cleaner separation from $P_{\mu e}\left(I H, \delta_{C P}\right)$ can be obtained only if $\delta_{C P}$ is in the LHP. If IH is the true hierarchy, $\hat{\Delta} \approx-90^{\circ}$. Then $P_{\mu e}$ is reduced, and moved away from $P_{\mu e}\left(N H, \delta_{C P}\right)$ if $\delta_{C P}$ is in the UHP. Thus UHP forms the favourable half plane for IH, whereas LHP is the favourable half plane for NH. Even if we use the anti-neutrino oscillation probabilities, the same considerations will hold. Therefore, the same relation between hierarchy and half-plane holds for both neutrino and anti-neutrino data.

\subsection{Hierarchy discrimination ability of NOvA}

We plot the hierarchy discrimination ability of NOvA with nominal and increased statistics in Fig. 1. We see that, for $\sin ^{2} 2 \theta_{13}=0.1$ and with nominal design, the hierarchy can be determined at $90 \%$ C.L. for the following two combinations: $\left(\mathrm{NH},-170^{\circ} \leq \delta_{C P} \leq-10^{\circ}\right)$ or $\left(\mathrm{IH}, 10^{\circ} \leq \delta_{C P} \leq\right.$ $170^{\circ}$ ). The statistics for the experiment are not quite enough to determine the hierarchy for the whole favourable half plane for this value of $\theta_{13}$. If $\sin ^{2} 2 \theta_{13}=0.12$, then the hierarchy can be determined for the whole favoured half plane. It was shown in Ref. [24] that NOvA can determine the hierarchy for $45 \%$ of the $\delta_{C P}$ range for $\sin ^{2} 2 \theta_{13}=0.1$.
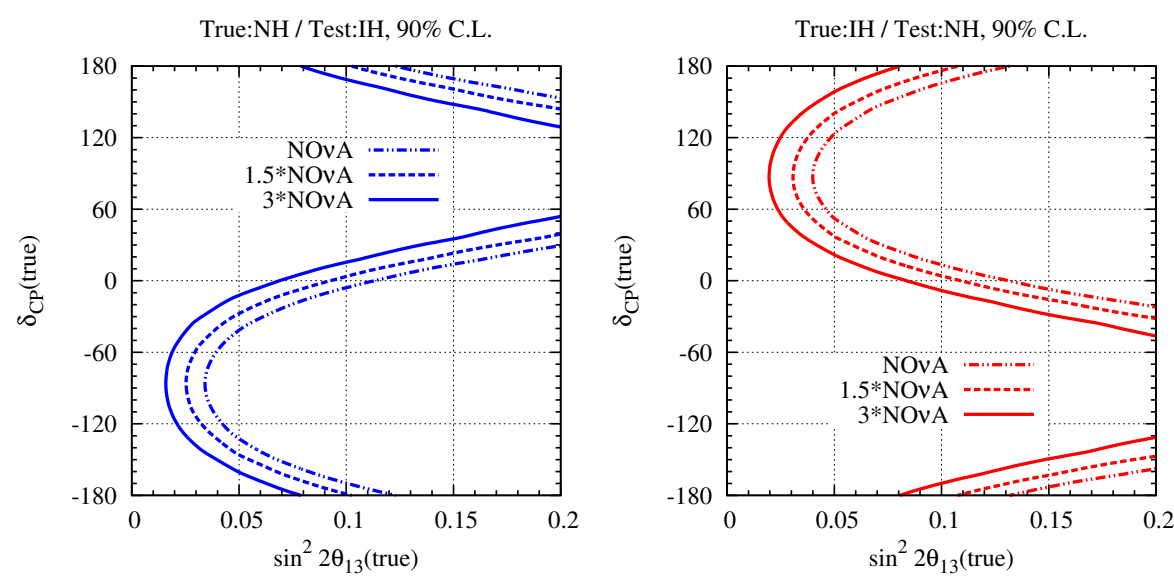

Figure 1: (colour online) Hierarchy exclusion plots for NOvA with boosted statistics for $3 v+3 \bar{v}$ running when NH is true (left panel) and when IH is true (right panel).

For smaller values of $\sin ^{2} 2 \theta_{13}$, one needs larger statistics to determine the hierarchy for the whole favourable half plane. With 1.5 times the presently projected statistics of NOvA, one can determine the hierarchy for the whole of the respective favourable half planes, for both $\mathrm{NH}$ and $\mathrm{IH}$, for $\sin ^{2} 2 \theta_{13}=0.1$. Similar conclusions were obtained earlier in Ref. [25]. If $\delta_{C P}$ happens to be in the unfavourable half plane, even tripling of statistics leads to hierarchy determination only for a very small range of $\delta_{C P}$.

\subsection{Hierarchy discrimination ability of NO $v \mathrm{~A}$ and T2K}

In this subsection, we explore how data from $\mathrm{T} 2 \mathrm{~K}$ can help in improving the sensitivity to hierarchy. We found that for $\sin ^{2} 2 \theta_{13} \leq 0.1$, hierarchy determination is not possible for any $\delta_{C P}$ in the unfavourable half-plane, if nominal design statistics of NOvA and T2K are assumed. Hence, we consider how hierarchy sensitivity improves with increasing statistics. We consider three scenarios:

- T2K will have a 5 year neutrino run with its design luminosity and NOvA will run according to its present plan. 
- T2K will have twice the above statistics and NOvA will have 1.5 times its designed statistics.

- T2K will have four times the above statistics and NOvA will have thrice its designed statistics.

The exclusion plots are given in Fig. 2. For all points to the right of the contours, the wrong hierarchy can be ruled out.
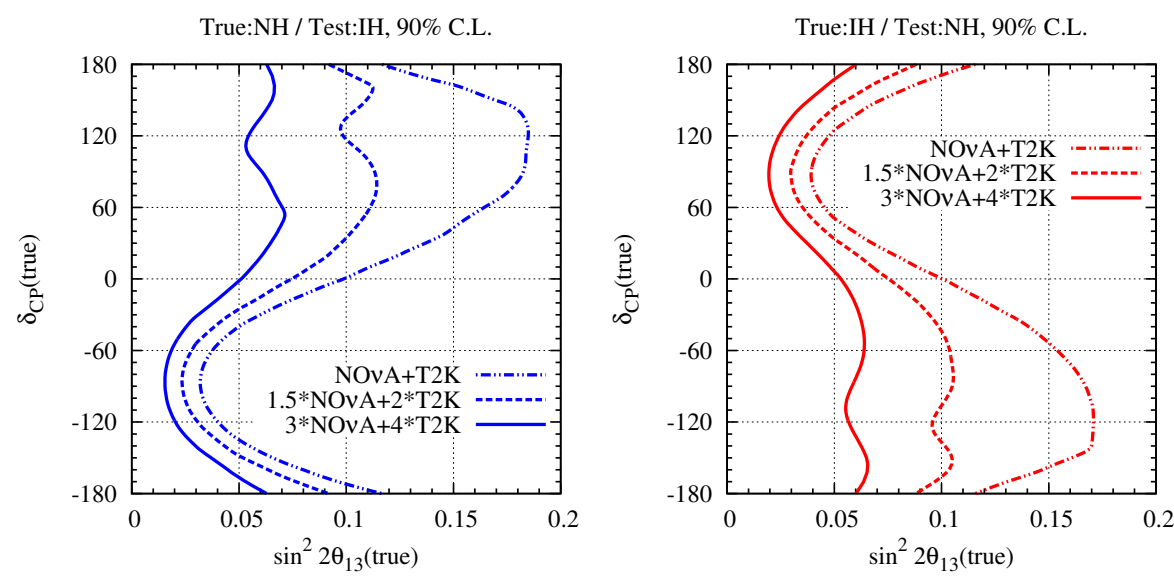

Figure 2: (colour online) Hierarchy exclusion plots for combined data from NOvA and T2K with various boosts in statistics when NH is true (left panel) and when IH is true (right panel).

In the left panel we assumed NH is the true hierarchy and in the right panel we assumed IH is the true hierarchy. We see that increasing the statistics from nominal values to $1.5^{*} \mathrm{NOVA}+2 * \mathrm{~T} 2 \mathrm{~K}$ dramatically improves the ability to rule out the wrong hierarchy, if $\delta_{C P}($ true $)$ is in the unfavourable half-plane. Further improvement occurs if the statistics are increased even more. In particular, if $\sin ^{2} 2 \theta_{13}=0.1[6,7]$, the hierarchy can be essentially established at $90 \%$ C.L., for any true value of $\delta_{C P}$, with 1.5 times the designed statistics of NOvA and twice the designed statistics of T2K. This point was noted previously in Ref. [25].

\section{Measuring $\delta_{C P}$ with $P_{\mu e}$}

In the following, we present 'allowed $\delta_{C P}$ ' graphs. In generating these, we have kept $\sin ^{2} 2 \theta_{13}$ fixed at 0.1 . The graphs are plotted in the true $\delta_{C P}$-test $\delta_{C P}$ plane. For every true value of $\delta_{C P}$, we indicate the range in test $\delta_{C P}$ that can be excluded at $90 \%$ C.L. The plots have been shown for both true and wrong hierarchies. The dotted range, defined by $\chi^{2} \leq 2.71$, shows the values of test $\delta_{C P}$ that are compatible with the data, generated with $\delta_{C P}$ (true) as input. For a given true value of $\delta_{C P}$, the error in measuring $\delta_{C P}$ is indicated by the spread of the dotted range along that $\delta_{C P}$ (true) vertical line.

If the statistics are increased to $1.5 * \mathrm{NOv} \mathrm{A}+2 * \mathrm{~T} 2 \mathrm{~K}$, as seen in Fig. 3 then most of the wrong hierarchy allowed region is ruled out as already noted in section 3. For the true hierarchy, the allowed region is centered around true $\delta_{C P}$ and is mostly in the correct half-plane. For the CP conserving case $\delta_{C P}=0\left(\delta_{C P}= \pm 180^{\circ}\right)$, there is a small additional allowed region around $\delta_{C P}=$ $\pm 180^{\circ}\left(\delta_{C P}=0\right)$ but for which $\chi^{2}$ is higher. If we limit our attention to the regions around $\chi_{\min }^{2}$, 
then $1.5^{*} \mathrm{NOvA}+2^{*} \mathrm{~T} 2 \mathrm{~K}$ can measure $\delta_{C P}$ with an accuracy of $\pm 40^{\circ}$ for true $\delta_{C P}=0$ and $\pm 60^{\circ}$ for true $\delta_{C P}= \pm 90^{\circ}$.

Thus we are led to the following important conclusion: $1.5 * \mathrm{NOv} \mathrm{A}+2 * \mathrm{~T} 2 \mathrm{~K}$ can essentially determine the hierarchy and also give an allowed region of $\delta_{C P}$ centered around its true value. Doubling of statistics will not lead to too much improvement in the allowed range of $\delta_{C P}$. Further strategies are needed to measure $\delta_{C P}$ to a good accuracy.
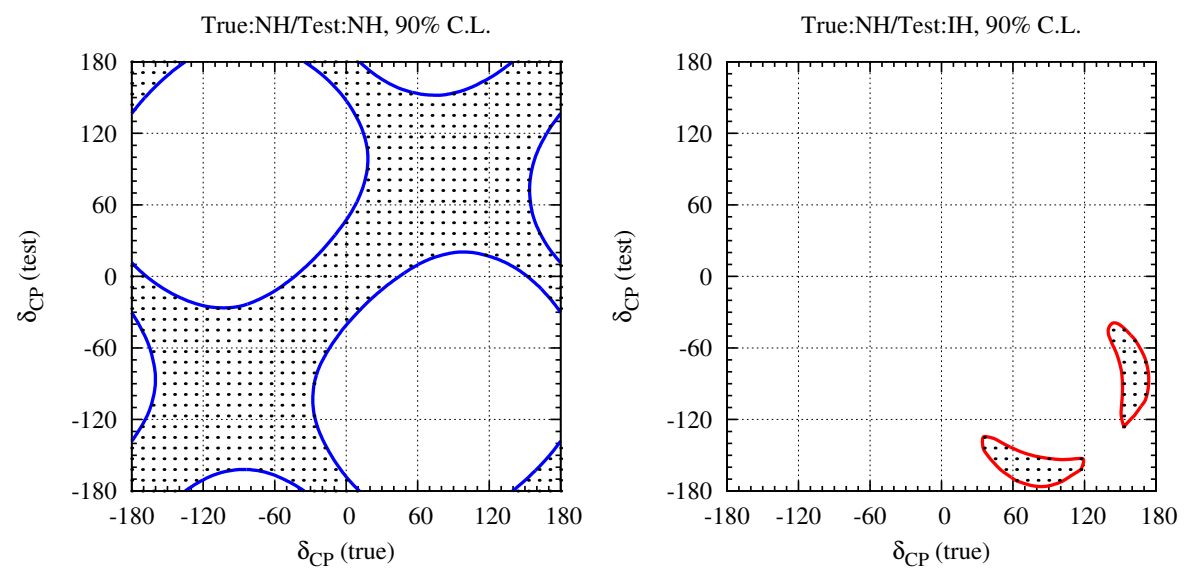

Figure 3: (colour online) Allowed $\delta_{C P}$ plots for $1.5 * \mathrm{NO} v \mathrm{~A}+2 * \mathrm{~T} 2 \mathrm{~K}$. Here $\mathrm{NH}$ is true. True and test $\sin ^{2} 2 \theta_{13}=0.1$. Test hierarchy is normal (left panel) and inverted (right panel).

\section{References}

[1] T2K Collaboration, K. Abe et al., Indication of Electron Neutrino Appearance from an Accelerator-produced Off-axis Muon Neutrino Beam, Phys. Rev. Lett. 107 (2011) 041801, [1106.2822].

[2] MINOS Collaboration Collaboration, P. Adamson et al., Improved search for muon-neutrino to electron-neutrino oscillations in MINOS, Phys. Rev. Lett. 107 (2011) 181802, [1108. 0015 ].

[3] Double Chooz Collaboration, Y. Abe et al., Indication for the disappearance of reactor electron antineutrinos in the Double Chooz experiment, Phys. Rev. Lett. 108 (2012) 131801, [1112.6353].

[4] Daya-Bay Collaboration Collaboration, F. An et al., Observation of electron-antineutrino disappearance at Daya Bay, Phys.Rev.Lett. 108 (2012) 171803, [1203.1669].

[5] RENO collaboration Collaboration, J. Ahn et al., Observation of Reactor Electron Antineutrino Disappearance in the RENO Experiment, Phys. Rev. Lett. 108 (2012) 191802, [1204. 0626 ].

[6] P. Machado, H. Minakata, H. Nunokawa, and R. Funchal, Combining Accelerator and Reactor Measurements of theta_13: The First Result, 1111.3330.

[7] G. Fogli, E. Lisi, A. Marrone, D. Montanino, A. Palazzo, et al., Global analysis of neutrino masses, mixings and phases: entering the era of leptonic CP violation searches, 1205.5254.

[8] S. Prakash, S. K. Raut, and S. U. Sankar, Getting the Best Out of T2K and NOvA, Phys.Rev. D86 (2012) 033012, [arXiv:1201.6485].

[9] P. Huber, M. Lindner, and W. Winter, Simulation of long-baseline neutrino oscillation experiments with GLoBES, Comput. Phys. Commun. 167 (2005) 195, [hep-ph/ 0407333 ]. 
[10] P. Huber, J. Kopp, M. Lindner, M. Rolinec, and W. Winter, New features in the simulation of neutrino oscillation experiments with GLoBES 3.0, Comput. Phys. Commun. 177 (2007) 432-438, [hep-ph/0701187].

[11] T2K Collaboration, Y. Itow et al., The JHF-Kamioka neutrino project, hep-ex/0106019.

[12] M. Ishitsuka, T. Kajita, H. Minakata, and H. Nunokawa, Resolving Neutrino Mass Hierarchy and CP Degeneracy by Two Identical Detectors with Different Baselines, Phys. Rev. D72 (2005) 033003, [hep-ph/0504026].

[13] P. Huber, M. Lindner, and W. Winter, Superbeams versus neutrino factories, Nucl.Phys. B645 (2002) 3-48, [hep-ph/0204352].

[14] NOvA Collaboration Collaboration, D. Ayres et al., NOvA: Proposal to build a 30 kiloton off-axis detector to study $n u(m u) \longrightarrow n u(e)$ oscillations in the NuMI beamline, hep-ex/0503053.

[15] NOvA Collaboration, T. Yang and S. Wojcicki, Study of physics sensitivity of $v \_m u$ disappearance in a totally active version of nova detector, $\mathrm{Off}-\mathrm{Axi} \mathrm{S}-\mathrm{Not} \mathrm{e}-\mathrm{SIM}-30$.

[16] M. D. Messier, Evidence for neutrino mass from observations of atmospheric neutrinos with Super-Kamiokande, . Ph.D. Thesis (Advisor: James L. Stone), UMI-99-23965.

[17] E. Paschos and J. Yu, Neutrino interactions in oscillation experiments, Phys.Rev. D65 (2002) 033002, [hep-ph/0107261].

[18] M. Fechner, Détermination des performances attendues sur la recherche de l'oscillation $v \_\mu \rightarrow v \_e$ dans l'expérience $T 2 K$ depuis l'étude des données recueillies dans l'expérience $K 2 K$, . Presented on 9 May 2006.

[19] T2K Collaboration Collaboration, I. Kato, Status of the T2K experiment, J.Phys.Conf.Ser. 136 (2008) 022018.

[20] A. Cervera et al., Golden measurements at a neutrino factory, Nucl. Phys. B579 (2000) 17-55, [hep-ph/0002108].

[21] E. K. Akhmedov, R. Johansson, M. Lindner, T. Ohlsson, and T. Schwetz, Series expansions for three-flavor neutrino oscillation probabilities in matter, JHEP 04 (2004) 078, [hep-ph / 0402175 ].

[22] M. Freund, Analytic approximations for three neutrino oscillation parameters and probabilities in matter, Phys. Rev. D64 (2001) 053003, [hep-ph/ 0103300$].$

[23] L. Wolfenstein, Neutrino oscillations in matter, Phys. Rev. D17 (1978) 2369-2374.

[24] P. Huber, M. Lindner, T. Schwetz, and W. Winter, First hint for CP violation in neutrino oscillations from upcoming superbeam and reactor experiments, JHEP 11 (2009) 044, [0 907 . 1896 ].

[25] NOvA Collaboration Collaboration, D. Ayres et al., The NOvA Technical Design Report, . FERMILAB-DESIGN-2007-01. 